Review

\title{
Protein folding at extreme temperatures: Current issues
}

\author{
Georges Feller \\ Laboratory of Biochemistry, Center for Protein Engineering-InBioS, University of Liège, Institute of Chemistry B6a, 4000 Liège-Sart Tilman, Belgium
}

\section{A R T I C L E I N F O}

\section{Article history:}

Received 25 February 2017

Received in revised form 18 August 2017

Accepted 5 September 2017

Available online 25 September 2017

\section{Keywords:}

Protein folding

Extremophiles

Trigger factor

Prolyl isomerization

Thermodynamic stability

\begin{abstract}
A B S T R A C T
The range of temperatures compatible with life is currently estimated from $-25^{\circ} \mathrm{C}$, as exemplified by metabolically active bacteria between sea ice crystals, and up to $122^{\circ} \mathrm{C}$ in hydrothermal vents as exemplified by the archaeon Methanopyrus kandleri. In the context of protein folding, as soon as a polypeptide emerges from the ribosome, it is exposed to the effects of environmental temperatures. Recent investigations have shown that the rate of protein folding is not adapted to extreme temperatures and should be very fast at high temperature and low in cold environments. This lack of adaptation is driven by kinetic constraints on protein stability. To counteract the deleterious effects of fast protein folding in hyperthermophiles, chaperones such as the Trigger Factor hold and slow down the rate of folding intermediates. Prolyl isomerization, a rate-limiting step in the folding of many proteins, is strongly temperature-dependent and impairs folding of psychrophilic proteins in the cold. This is compensated by reduction of the proline content in cold-adapted proteins, by an increased number of prolyl isomerases encoded in the genome of psychrophilic microorganisms and by overexpression of prolyl isomerases under low temperature cultivation. After folding, the native state is reached and although extremophilic proteins share the same fold, dramatic differences in stability have been recorded by differential scanning calorimetry.
\end{abstract}

(c) 2017 Elsevier Ltd. All rights reserved.

\section{Contents}

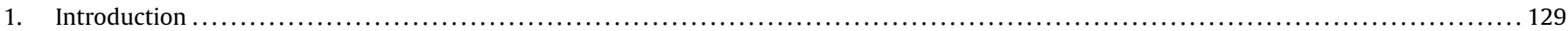

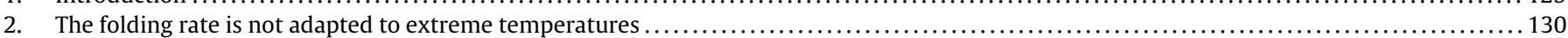

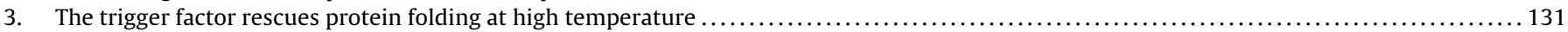

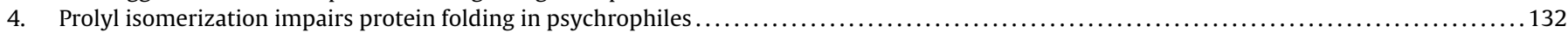

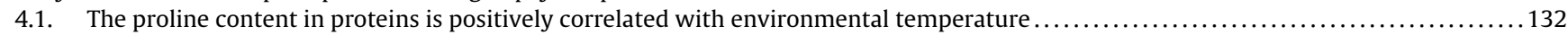

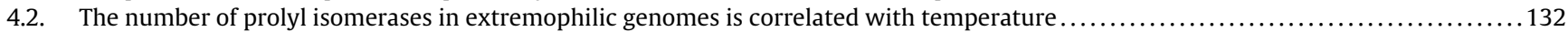

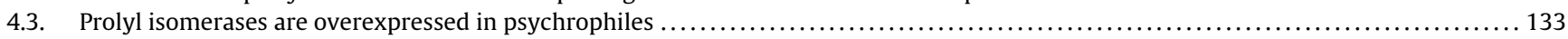

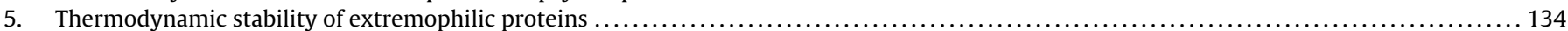

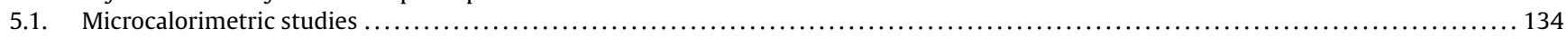

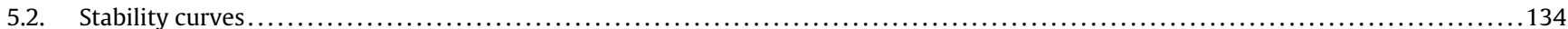

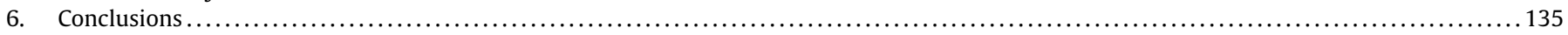

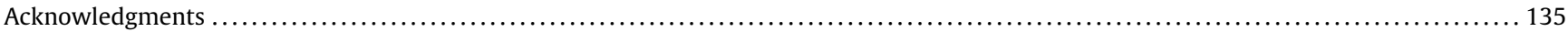

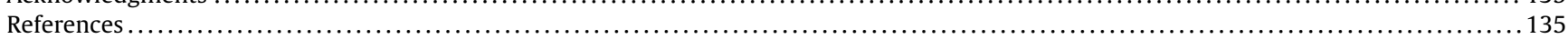

\section{Introduction}

In recent years, microbiological investigations of environments previously regarded as abiotic have considerably expanded the

\footnotetext{
Abbreviations: TF, trigger factor; PPlase, prolyl isomerase or peptidyl-prolyl cis/trans isomerase; GFP, green fluorescent protein; DSC, differential scanning calorimetry.

E-mail address: gfeller@ulg.ac.be
}

spectrum of biological temperatures. Metabolically active bacteria have been found at $-20^{\circ} \mathrm{C}$, thriving in the liquid brine veins between sea ice crystals $[1,2]$. More recently, the bacterium Planococcus halocryophilus isolated from Arctic permafrost was found to grow and to divide at $-15^{\circ} \mathrm{C}$ and to display residual metabolic activity at $-25^{\circ} \mathrm{C}$ [3], which possibly represents the lower temperature limit before dormancy. These low temperature extremophiles are termed psychrophiles. At the other extremity of the temperature range compatible with life, thermophiles are known for decades but hyperthermophiles have pushed the 
limit above $100^{\circ} \mathrm{C}$. Hyperthermophilic bacteria such as Thermotoga maritima [4] or Aquifex aeolicus [5] isolated from geothermally heated seafloor sediments are limited to $90-95^{\circ} \mathrm{C}$, whereas the most extreme environments such as deep sea hydrothermal vents, are exclusively colonized by Archaea. For many years, the archaeon Pyrolobus fumarii growing at $113^{\circ} \mathrm{C}$ was regarded as the most heat-resistant microorganism [6], but more recently Methanopyrus kandleri, thriving at $122^{\circ} \mathrm{C}$ and enduring exposure up to $130^{\circ} \mathrm{C}$, has established the current upper temperature limit for life [7]. It should be noted that such high temperature for cell proliferation is reached under in situ high pressure.

Microbial life under these extreme environmental temperatures obviously requires a vast array of adaptations at all cellular levels, including macromolecules. In this respect, proteins and enzymes play a pivotal role as they drive the cell cycle and metabolism. At low temperature, enzymes have to remain active and proteins have to maintain adequate functional dynamics at temperatures that slow down molecular motions. This is reached by adopting a mobile and flexible structure through reduction of all types of weak interactions involved in the shaping of protein conformation such as hydrogen bonds, salt bridges, van der Waals contacts, helix dipole, etc. and the hydrophobic effect. But the price to pay for such an improved structural dynamics is the pronounced heat-lability of psychrophilic proteins [8-12]. In contrast, thermophilic and hyperthermophilic proteins have to maintain a functional native state at elevated temperatures that would otherwise unfold mesophilic proteins. Their robust and heat-stable structure arises from the improvement in strength and number of all types of weak interactions and structural factors stabilizing the protein conformation [13-15].

Here, we will focus on protein folding, a crucial topic for the biophysical understanding of life at extreme temperatures. Indeed, as soon as a nascent polypeptide emerges from the ribosome, it is exposed to the effects of environmental temperatures. Recent investigations have addressed some essential questions: $i$ ) what is the effect of extreme environmental temperatures on the protein folding rate; $i$ ) the Trigger Factor (TF) is the first chaperone interacting with nascent chains: how does it help protein folding at extreme temperatures; iii) how do prolyl isomerases catalyze prolyl isomerization, a rate-limiting step in protein folding and $i v$ ) what are the properties of the final native state of protein adapted to these temperatures? Many of the reviewed results have been obtained with TF as a model protein. In Bacteria, TF is a ribosome-bound chaperone interacting with virtually all synthesized polypeptides and it also possesses a peptidyl-prolyl cis/trans isomerase (PPiase) activity [16]. This chaperone has been isolated from model bacteria: the Antarctic psychrophile Pseudoalteromonas haloplanktis, a fast growing strain at low temperature [17], the mesophilic reference Escherichia coli and the hyperthermophile Thermotoga maritima [4].

\section{The folding rate is not adapted to extreme temperatures}

In order to determine the folding rate constant, the unfolded protein (usually in urea or guanidinium chloride) is diluted with a buffer and its refolding is monitored by a stopped-flow spectrophotometer as the process is completed within a few seconds. Similar experiments are performed for unfolding, starting with the native state (Fig. 1).

By using a range of final denaturant concentrations, a "Chevron plot" is constructed (see Fig. 2 as an example).

The left arm of the plot corresponds to folding kinetics and the right arm depicts unfolding kinetics. Finally, the most relevant parameters, the microscopic rate constants for folding $k_{f}\left(\mathrm{H}_{2} \mathrm{O}\right)$ and for unfolding $k_{u}\left(\mathrm{H}_{2} \mathrm{O}\right)$ are obtained by extrapolation on the $\mathrm{Y}$ axis, i.e. in the absence of denaturant, in $\mathrm{H}_{2} \mathrm{O}$.

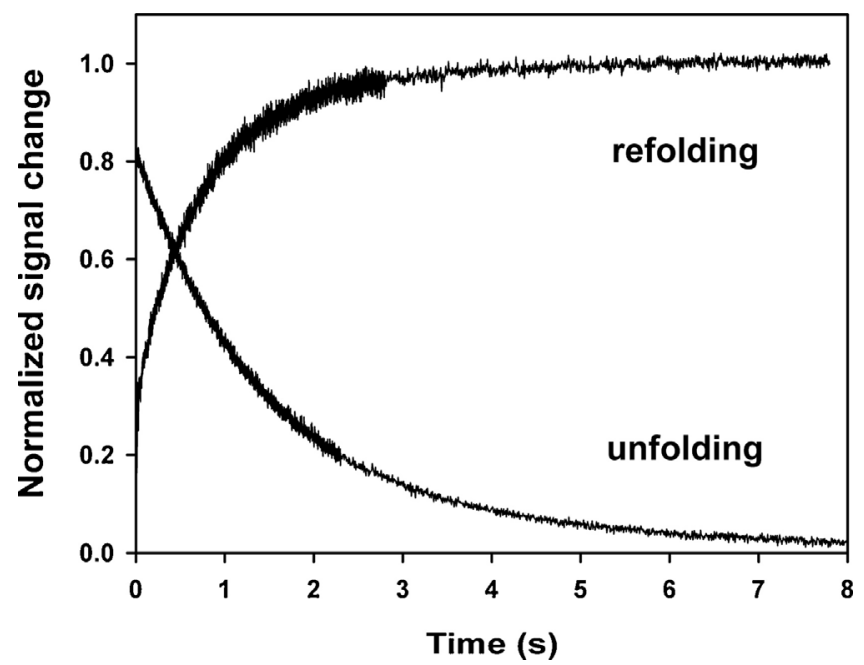

Fig. 1. Representative folding and unfolding fluorescence traces recorded by stopped-flow. Rate constants are obtained by adjusting one or several exponential functions to the kinetic traces. Unpublished data from [18] using a psychrophilic TF.

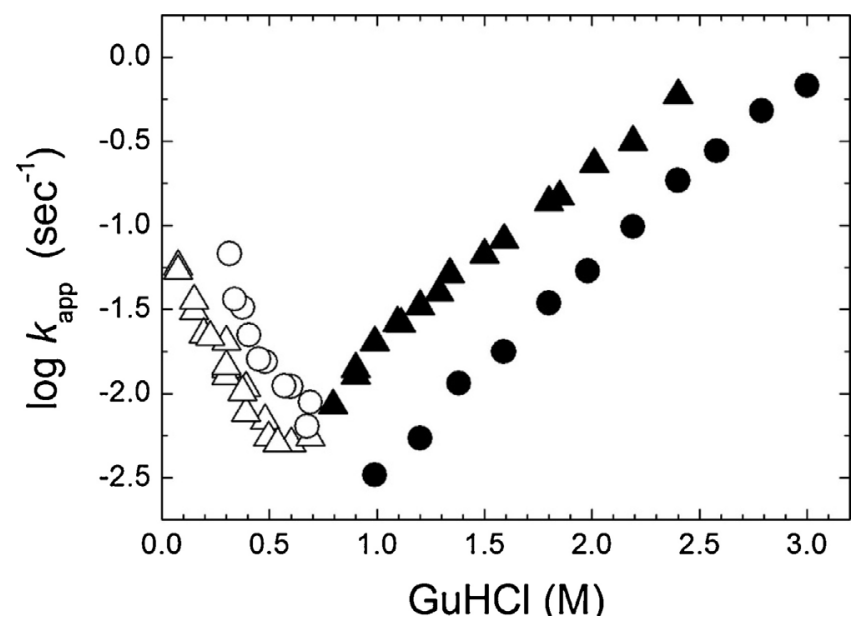

Fig. 2. Chevron plots of the rate constants $k_{a p p}$ for unfolding (closed symbols, right arms) and refolding (open symbols, left arms) of tryptophan synthase $\alpha$ subunit from the psychrophile $S$. frigidimarina $(\boldsymbol{\Lambda}, \triangle)$ and from the mesophile $\operatorname{E}$. coli $(\bullet, \bigcirc)$ at $25^{\circ} \mathrm{C}$.

Reprinted from [19] by permission of Oxford University Press.

Because these rate constants strongly differ in proteins and are also affected by the experimental setup, it is essential to compare series of homologous extremophilic proteins under the same experimental conditions [20]. This has been performed using the above-mentioned TF [18]. For the sake of clarity, Fig. 3 is a simplified version of the original work (only the main phases are depicted) that allows one to draw several conclusions. As far as folding is concerned, at the same temperature, the psychrophilic TF (Fig. 3, left panel, blue line) folds slower than its mesophilic homologue (black line) and the hyperthermophilic protein (red line) folds slightly faster. In the narrow window of temperature accessible to stoppedflow experiments, the temperature dependence of this folding rate was similar for the three proteins. Accordingly, one has to conclude that the folding rate is not adapted to extreme temperatures because under environmental conditions the hyperthermophilic protein should fold extremely fast, whereas the psychrophilic protein should fold even slower in the cold. The same observation was made for a psychrophilic tryptophan synthase subunit (Fig. 2) as compared with its E. coli homologue [19] and suggests a common behavior of cold-adapted proteins. On the other hand, proteins from 


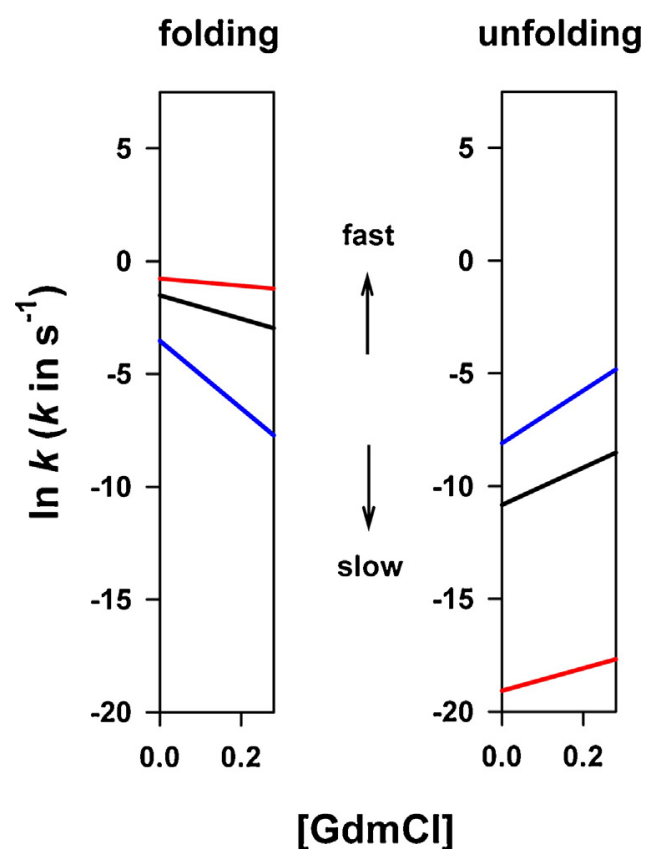

Fig. 3. Close-up of ordinate extrapolations from Chevron-plots. Data are shown for psychrophilic (blue), mesophilic (black) and hyperthermophilic (red) TFs at $9{ }^{\circ} \mathrm{C}$. Extrapolations of the rate constants for the determination of the microscopic rate constants for folding $k_{f}\left(\mathrm{H}_{2} \mathrm{O}\right)$ and unfolding $k_{u}\left(\mathrm{H}_{2} \mathrm{O}\right)$ in the absence of denaturant. Adapted from [18].

thermophiles and hyperthermophiles generally fold slightly faster [21-23] or with similar rate constants [21,24-26] as compared with their mesophilic homologues.

In contrast to folding, the differences in the unfolding rates of extremophilic TF are much larger (Fig. 3, right panel). The hyperthermophilic protein unfolds very slowly, whereas the psychrophilic protein unfolds faster as compared with the mesophilic counterpart. In fact, the slow unfolding of hyperthermophilic proteins has been previously identified as the main kinetic contribution to the unusually high stability of these proteins [24-29]. Indeed, in a simple two-state equilibrium between the unfolded state $\mathrm{U}$ and the native state $\mathrm{N}$ shown in Eq. (1),

$\mathrm{U} \underset{k_{u}}{\stackrel{k_{f}}{\rightleftharpoons}} \mathrm{N}$

a forward displacement of the equilibrium towards $\mathrm{N}$, i.e. an increase of stability, is obtained by a slower unfolding rate $k_{u}$, as observed in heat-stable proteins. Conversely, a backward displacement of the equilibrium, i.e. a decrease of stability, is reached by a faster unfolding rate $k_{u}$ and this is precisely what is observed for the natively unstable psychrophilic proteins (Figs. 2 and 3) $[18,19]$ and also predicted by molecular dynamics simulations [30]. Very significantly, stabilization of a psychrophilic alpha-amylase by site-directed mutagenesis results in a slower unfolding rate of the mutants [31], underlining the kinetic strategy leading to the natively unstable conformation of psychrophilic proteins. Therefore, there is a fine tuning of the unfolding rate values in order to adjust protein stability to the environmental temperature. This results in a continuum from fast to slow unfolding rates in unstable to hyperstable proteins.

However, the same rationale can be applied to the folding rate $k_{f}$. In Eq. (1), an increase in stability is obtained by a fast folding rate $k_{f}$, whereas a lower stability is reached by a slower folding rate. Again, this is exactly what is observed for hyperthermophilic and psychrophilic proteins, respectively (Figs. 2 and 3). Significantly also, all mutants of a hyperthermophilic enzyme destabilized

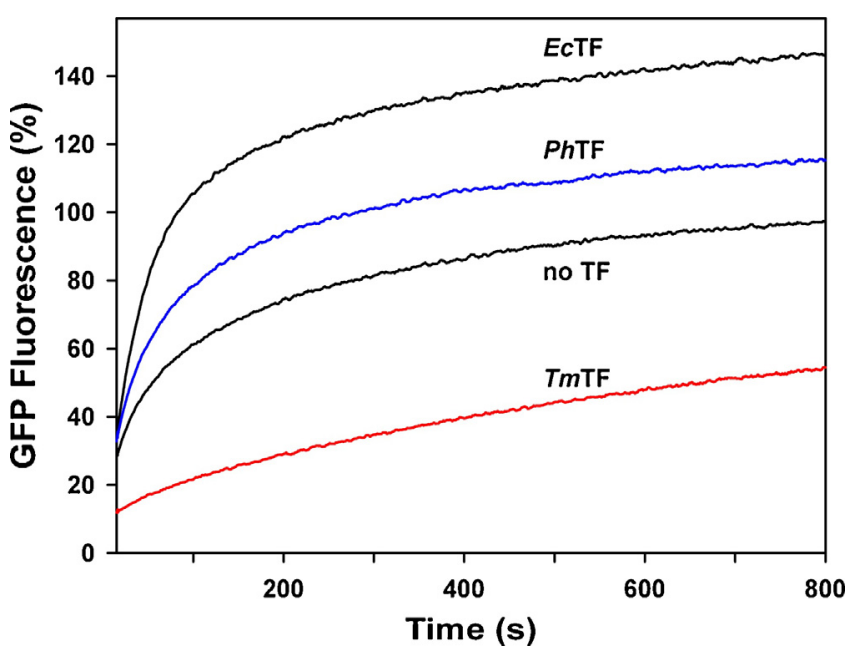

Fig. 4. GFP refolding assisted by trigger factors. Data are shown for psychrophilic (blue), mesophilic (black) and hyperthermophilic (red) TFs. Refolding time courses of acid-denatured GFP at $15^{\circ} \mathrm{C}$ recorded by fluorescence. Fluorescence intensity (extrapolated to the infinite) of spontaneously refolded GFP (no TF) is taken as $100 \%$. Adapted from [33].

by site-directed mutagenesis displayed both faster unfolding rates and slower folding rates than the wild-type protein [25,32]. In summary, the natively unstable structure of psychrophilic proteins is gained via both a fast unfolding rate and a slow folding rate. Conversely, hyperthermophilic proteins are characterized by a slow unfolding rate and, to a lower extent, by a fast or unchanged folding rate. It follows that the folding rate cannot be adapted to extreme temperatures because adjustments of protein stability to environmental temperature is under kinetic control. Lowering the folding rate in hyperthermophiles would result in protein destabilization, whereas accelerating the folding rate in psychrophiles would increase protein stability.

What are the physiological consequences for the nascent polypeptide? Intuitively, the slow folding rate of psychrophilic proteins should not be a concern, except that it contributes to slow down the cell cycle, as observed in the environment but resulting from many other temperature sensitive cellular events. In contrast, both the fast folding rate of hyperthermophilic proteins and high temperature should have deleterious effects for the nascent polypeptide because they increase the probability of misfolding events and aggregation of misfolded species. This aspect is analyzed in the next section.

\section{The trigger factor rescues protein folding at high temperature}

If the folding rate is not adapted to extreme temperatures in the test tube, chaperones can have a pivotal role to rescue protein folding in vivo. TF is the first folding assistant acting co-translationally on synthesized polypeptides in bacteria. This is an obvious candidate to test the effects of chaperones and this has been performed using the above-mentioned extremophilic TFs [33]. Fig. 4 illustrates a classical refolding experiment in which acid denatured green fluorescent protein (GFP) is allowed to refold after buffer neutralization, either alone or in the presence of added TF. The fluorescence intensity of GFP is directly proportional to the concentration of native GFP in the experiment. The mesophilic TF from E. coli is a very efficient foldase that can improve the yield of native GFP up to $150 \%$. The psychrophilic TF is also a foldase with however a weaker chaperone activity. This can be tentatively related to the fact that low temperature slows down protein folding and reduces the probability of misfolding and aggregation. As a matter of fact, low 


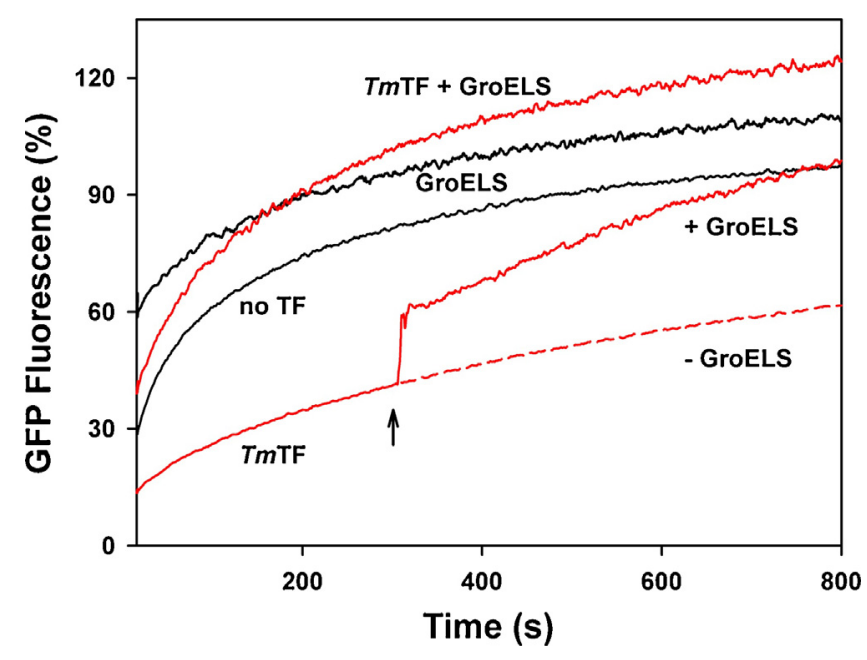

Fig. 5. GFP refolding in the presence of TF from the hyperthermophile T. maritima and GroELS at $15^{\circ} \mathrm{C}$. Fluorescence time courses of GFP alone and in the presence of GroELS, TmTF or TmTF+ GroELS. In the sequential experiment, addition of GroELS after $300 \mathrm{~s}$ is indicated by an arrow.

Adapted from [33].
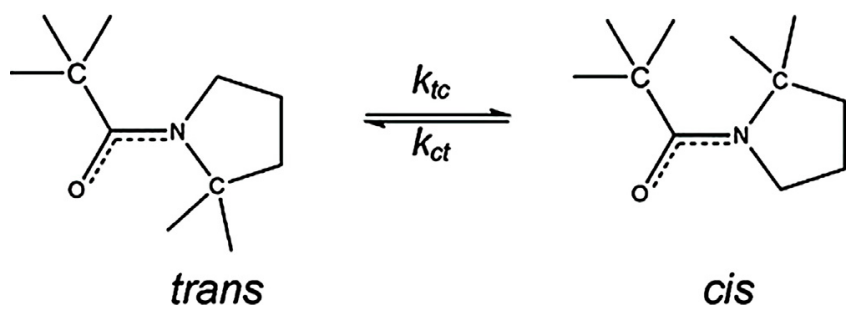

Fig. 6. Trans and cis isomers of a peptidyl-prolyl peptide bond. Reprinted with permission from [41]. Copyright 2009 American Chemical Society.

temperature cultivation of $E$. coli frequently avoids the formation of insoluble inclusion bodies of recombinant proteins [34], as well as expression in a psychrophilic host at low temperature [35]. Furthermore, low temperature weakens the hydrophobic effect. Therefore, an efficient foldase is possibly not required by psychrophiles in cold conditions. But the most insightful result was obtained with the hyperthermophilic TF. As shown in Fig. 4, TF from T. maritima is not a foldase (it does not promote the spontaneous GFP refolding) but instead it is a holdase: it binds to the refolding GFP and drastically decreases its folding rate. Accordingly, the holdase function of this TF should be regarded as the main adaptation in hyperthermophilic bacteria in order to counteract the deleterious effects of high temperature on protein folding.

Interestingly, when the well-known GroELS chaperone is added to the refolding mixture, a burst in refolded GFP is observed (Fig. 5). A similar result has been reported for the thermophilic TF from Thermus thermophilus [36]. This strongly suggests that the hyperthermophilic TF acts as a carrier of folding intermediates for delivery to downstream chaperones and final maturation. Furthermore, the strongly improved yield of native GFP in the presence of both TF and GroELS suggests that the hyperthermophilic TF maintains intermediate species in a folding-competent state which favors the action of downstream chaperones.

To explore the holdase function, ANS titration and isothermal titration calorimetry have revealed a hydrophobic chaperone cavity which potentially binds apolar components of folding intermediates, therefore lowering their rate of internalization and consequently the folding rate of the client protein [33]. The stoichiometry of interaction with $\alpha$-casein, an intrinsically disordered protein (natively unfolded), indicated a higher number of hyperthermophilic TF molecules bound to the unfolded state as compared with the mesophilic homologue, which also contributes to the strong holdase activity.

Further studies are awaited in order to unravel the complex interplay between nascent polypeptides and chaperones at high temperature. This is especially relevant for hyperthermophilic archaea which possess a specific chaperone network, distinct from that found in bacteria $[37,38]$.

\section{Prolyl isomerization impairs protein folding in psychrophiles}

Prolyl isomerization is a rate-limiting step in the folding of many proteins [39]. This arises from the weak steric constraints exerted by the pyrrolidine ring of proline to favor either the cis or the trans conformation in the unfolded state and to the slow isomerization involving the rotation about the peptide bond, which has a partial double-bond character (Fig. 6). It follows that the time spent by folding intermediates to explore the conformational space and to adopt the required prolyl cis or trans conformation is longer than for other amino acid side chains which are almost invariably constrained in trans. Prolyl isomerization intrinsically possesses a high activation energy and is therefore strongly temperature dependent [40]. In the context of extreme temperatures, one can intuitively assume that prolyl isomerization is very fast in hyperthermophiles and should not be a concern for protein folding. In contrast, slow prolyl isomerization at low temperature should impair the folding of psychrophilic proteins. Several lines of evidence indicate that this is indeed the actual situation.

\subsection{The proline content in proteins is positively correlated with environmental temperature}

The first observation refers to the proline content which is low in psychrophilic proteins and high in heat-stable polypeptides. For instance, in homologous alpha-amylases ( $\sim 50 \mathrm{kDa})$ the psychrophilic enzyme contains 13 prolines, the mesophilic homologue has 19 prolines and the heat-stable enzyme has 25 prolines, i.e. nearly twice the content of the cold-adapted protein. These differences have been related to adjustments of protein stability $[8,10,11]$. Indeed, in the proline residue, the structure of the pyrrolidine ring bonded to the main chain nitrogen locks a dihedral angle and severely restricts rotations of the backbone. This local rigidity induced by the prolyl residue is a well-known structural factor improving protein stability $[14,15,42]$. Consequently, heat-stable proteins display a high proline content, whereas psychrophilic proteins tend to decrease this content in order to reach a natively unstable conformation. In the context of protein folding, the high proline content of hyperthermophilic proteins should not be a concern as a result of fast prolyl isomerization at high temperature, whereas the low proline content of psychrophilic proteins reduces the probability of slow and rate-limiting folding steps. Therefore, the low proline content in psychrophilic polypeptides has two distinct contributions: it avoids prolyl-limited folding events and it destabilizes the native state.

\subsection{The number of prolyl isomerases in extremophilic genomes is correlated with temperature}

Cells are equipped with specialized catalysts, peptidyl-prolyl cis/trans isomerases (PPiases or rotamases), which speed-up prolyl isomerization in proteins. Fig. 7 illustrates a classical experiment in which increasing amounts of PPiase proportionally accelerate the folding of a proline-limited protein, revealing a true enzymatic activity [43]. The second observation indicating 


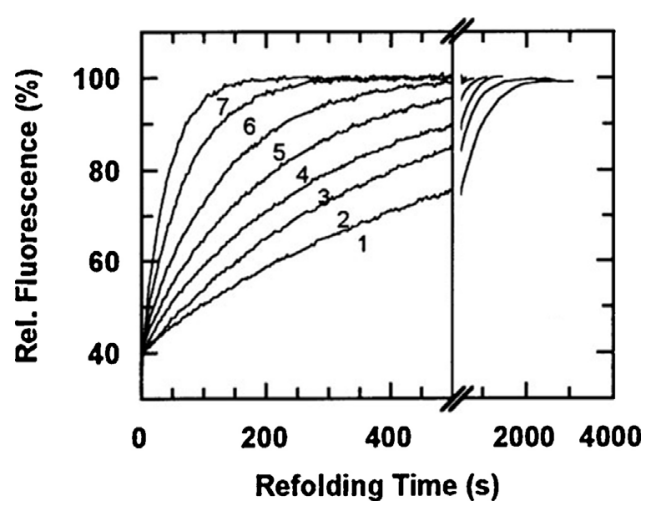

Fig. 7. Catalytic activity of PPiases on protein folding. The refolding rate of a model protein (RCM-T1, reduced and carboxymethylated ribonuclease T1) limited by proline isomerization can be slow (1). Addition of increased amounts of PPiase (2-7) accelerates the folding rate of the model protein, as shown by the increases of fluorescence.

Reprinted from [43] by permission from Macmillan Publishers: The EMBO Journal. Copyright 1997.

Table 1

Prolyl isomerases encoded in the genome of representative extremophilic and mesophilic Gram-negative bacteria and their environmental temperatures.

\begin{tabular}{lll}
\hline Strain & Temperature & PPiase genes \\
\hline Colwellia psychrerythraea $34 \mathrm{H}$ & $<0^{\circ} \mathrm{C}$ & 18 \\
Pseudolateromonas haloplanktis TAC 125 & $<0^{\circ} \mathrm{C}$ & 15 \\
Pseudomonas extremaustralis $14-3$ & $<0^{\circ} \mathrm{C}$ & 14 \\
Escherichia coli K12 & $37^{\circ} \mathrm{C}$ & 10 \\
Thermus thermophilus HB8/HB27 & $65{ }^{\circ} \mathrm{C}$ & 4 \\
Thermotoga maritima MSB8 & $85-90^{\circ} \mathrm{C}$ & 1 \\
Aquifex aeolicus VF5 & $85-95^{\circ} \mathrm{C}$ & 1 \\
\hline
\end{tabular}

that prolyl isomerization is influenced by extreme temperatures is the number of genes encoding PPiases in extremophilic genomes. Various types of PPiases are expressed in cells (FKBP-type, cyclophilin-type, parvulin-type) and all have been screened in representative extremophilic genomes using MaGe (www.genoscope.cns.fr/agc/microscope/home/index.php).

Table 1 shows that when compared with E. coli, the Gramnegative mesophilic reference, psychrophilic genomes contain a higher number of PPiase genes, nearly twice for C. psychrerythrae. Conversely, when the growth temperature increases, the number of PPiases gradually drops to 4 genes at $65^{\circ} \mathrm{C}$ and to 1 gene at $85-95^{\circ} \mathrm{C}$. Gram-positive bacteria encode a lower number of PPiases but the same trend is observed. The strains Planococcus antarcticus and $P$. halocryophilus thriving around $0{ }^{\circ} \mathrm{C}$ encode 7 and 6 PPiases, respectively, whereas the mesophiles $B$. subtilis and Enterococcus species all contain 4 PPiases. In contrast Geobacillus and Thermincola species thriving at $60^{\circ} \mathrm{C}$ only contain 3 PPiases. But the most convincing evidence that the PPiase genomic content is correlated to temperature is provided by methanogenic archaea because they have colonized the largest range of environmental temperatures. As shown in Table 2, mesophilic archaea encode 4-5 PPiases, whereas in the $65-85^{\circ} \mathrm{C}$ range only 2 PPiases are found. Significantly, $M$. kandleri, the most heat-resistant organism known to date, only encodes one PPiase. In addition, the genome of most hyperthermophilic archaea such as Pyrococcus furiosus, Sulfolobus solfataricus or Thermococcus species also only encodes one PPiase.

Overall, this analysis is a strong indication that prolyl isomerization is sufficiently fast at high temperature, whereas in psychrophiles this isomerization requires powerful catalytic assistance by a full set of PPiases. Furthermore, the persistence of only one PPiase in hyperthermophilic archaea suggests that the folding of a subset of essential proteins is limited by prolyl isomerization. Alternatively, other functions of archaeal PPiases, such as
Table 2

Prolyl isomerases encoded in the genome of representative extremophilic and mesophilic methanogenic archaea and their environmental temperatures.

\begin{tabular}{lll}
\hline Strain & Temperature & PPiase genes \\
\hline Methanosarcina barkeri & $25^{\circ} \mathrm{C}$ & 5 \\
Methanosarcina acetivorans & $37^{\circ} \mathrm{C}$ & 5 \\
Methanosarcina mazei & $37^{\circ} \mathrm{C}$ & 5 \\
Methanococcus maripaludis & $37^{\circ} \mathrm{C}$ & 4 \\
Methanococcus aeolicus & $45-50^{\circ} \mathrm{C}$ & 3 \\
Methanothermobacter thermautotrophicus & $65-70^{\circ} \mathrm{C}$ & 2 \\
Methanocaldococcus jannaschii & $85^{\circ} \mathrm{C}$ & 2 \\
Methanopyrus kandleri & $122^{\circ} \mathrm{C}$ & 1 \\
\hline
\end{tabular}

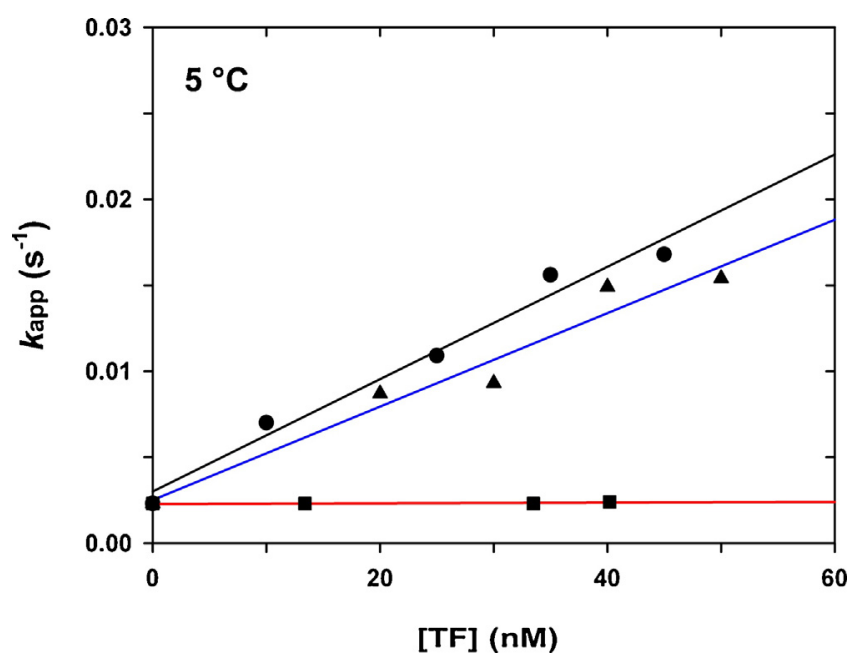

Fig. 8. Catalysis of refolding of RCM-T1 (reduced and carboxymethylated ribonuclease T1). Data for the psychrophilic TF ( $\mathbf{\Delta}$, blue), E. coli TF $(\bullet$, black) and the hyperthermophilic TF ( $\mathbf{\square}$, red). The apparent rate constant of prolyl isomerization during refolding of RCM-T1 is plotted as function of the TF concentration. Adapted from [33].

chaperone or anti-aggregation activities, could be responsible for their genomic persistence [44]. It should be mentioned that the above analysis depicts a general trend but not an absolute rule as exceptions occur in the database. This analysis strongly relies on the quality of genome annotations and other factors, such as the microorganism life style (salinity, pressure, ecological niche. . .), are possibly involved.

\subsection{Prolyl isomerases are overexpressed in psychrophiles}

Enzyme activity displays typical adaptive traits to environmental temperature $[8,45]$ and it was therefore of interest to check PPiases activity in extremophiles. There is only one PPiase shared by model extremophilic bacteria and this is the TF again. The PPiase activity was analyzed using newly developed substrates and methodologies [33]. Fig. 8 depicts such an experiment in which the increase of the folding rate of a proline-limited protein substrate is plotted as a function of the PPiase concentration in order to calculate the catalytic efficiency $k_{c a t} / K_{m}$ of the isomerization reaction. It can be seen that the PPiase activity of the hyperthermophilic protein is extremely low and close to the detection limit. This was expected because hyperthermophilic enzymes require high temperature to become fully activated and their rigid structure precludes significant activity, even at room temperature. More surprising was the same PPiase activity shared by both the psychrophilic and mesophilic TFs. Indeed, in most cases the activity of psychrophilic enzymes is very high in order to compensate for the decrease of reaction rates inherent to low temperature [10-12]. The psychrophilic PPiase escapes this rule but the reasons 


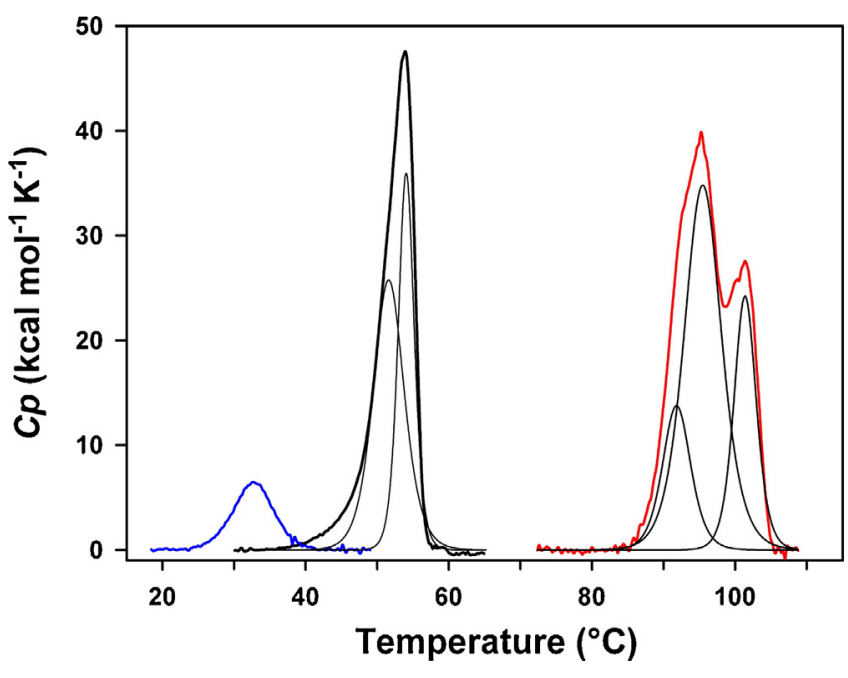

Fig. 9. Thermal unfolding of extremophilic proteins. Thermograms of DNA-ligases recorded by differential scanning microcalorimetry showing, from left to right, psychrophilic (blue), mesophilic (black) and hyperthermophilic (red) proteins. Adapted from [52].

remain unclear. This can be tentatively related to the peculiar but still hypothetical reaction mechanism of PPiases [46] which possibly precludes cold adaptation. However, proteomic studies have brought an unsuspected answer to this paradox [47]. Indeed, it was found that this TF is overexpressed nearly 40 times by the psychrophile $P$. haloplanktis under low temperature cultivation. Apparently, if the PPiase specific activity cannot be improved at low temperature, the microorganism adapts by dramatically increasing the enzyme concentration and therefore the available cellular PPiase activity. Moreover, overexpression of PPiases has been reported in almost all proteomic studies of psychrophiles so far [48] and this is probably the most firmly established result shared by proteomics of psychrophiles. Again, this points to the negative effect of prolyl isomerization on folding of psychrophilic proteins and to the requirement of catalytic assistance by PPiases.

\section{Thermodynamic stability of extremophilic proteins}

After all folding events, the native state is reached and although extremophilic proteins share the same fold and 3D structures, they display dramatic differences in terms of stability. The energetics of structure stability was essentially analyzed by differential scanning calorimetry (DSC) of homologous extremophilic proteins $[18,45,49-53]$.

\subsection{Microcalorimetric studies}

A demonstrative example of microcalorimetric records for the heat-induced unfolding of extremophilic proteins is illustrated in Fig. 9. These proteins clearly show distinct stability patterns that evolve from a simple unfolding profile in the unstable psychrophilic protein to a more complex profile in the stable hyperthermophilic counterpart. The unfolding of the cold-adapted protein occurs at much lower temperatures as indicated by the temperature of halfdenaturation $T_{m}=33^{\circ} \mathrm{C}$, given by the top of the transition (Table 3 ). Accordingly, this protein spontaneously unfolds at a mesophilic temperature of $37^{\circ} \mathrm{C}$. By contrast, the hyperthermophilic protein unfolds around $100^{\circ} \mathrm{C}$. Melting point values up to $150^{\circ} \mathrm{C}$ have been reported for hyperthermophilic archaeal proteins [54,55]. The calorimetric enthalpy $\Delta H_{\text {cal }}$ (area under the curves in Fig. 9) corresponds to the total amount of heat absorbed during unfolding, but it also reflects the enthalpy of disruption of bonds involved in
Table 3

Microcalorimetric parameters of thermal unfolding shown in Fig. 9.

\begin{tabular}{lll}
\hline DNA-ligases & $T_{m}\left({ }^{\circ} \mathrm{C}\right)$ & $\Delta H_{\text {cal }}\left(\mathrm{kcal} \mathrm{mol}^{-1}\right)$ \\
\hline Psychrophilic & 33 & 46 \\
Mesophilic & 54 & 253 \\
Hyperthermophilic & $95-101$ & 413 \\
\hline
\end{tabular}

maintaining the protein compact structure. There is a clear trend for increasing $\Delta H_{\text {cal }}$ values in the order psychrophile $<$ mesophile $<$ hyperthermophile (Table 3). On this basis, the hyperthermophilic protein possesses a nearly ten times higher stability provided by its various enthalpy-driven weak interactions when compared with the cold-adapted one. The psychrophilic protein unfolds according to a cooperative, all-or-none process, revealing a uniformly low stability of its architecture. By contrast, both homologous proteins display two to three transitions (indicated by deconvolution of the heat capacity function in Fig. 9). Therefore, the conformation of these mesophilic and hyperthermophilic proteins contains structural blocks or units of distinct stability that unfold independently.

From these observations, it can be concluded that psychrophilic proteins possess a fragile molecular edifice that is uniformly unstable and stabilized by fewer weak interactions than homologous mesophilic proteins. By contrast, hyperthermophilic proteins are robust molecules, made of various stability domains and stabilized by a high number of enthalpy-driven weak interactions.

\subsection{Stability curves}

The thermodynamic stability of a protein that unfolds reversibly according to a two-state mechanism (between the native state $\mathrm{N}$ and the unfolded state $\mathrm{U}$ ) is described by the classical GibbsHelmholtz relation:

$\Delta G_{N-U}=\Delta H_{N-U}-\mathrm{T} \Delta S_{N-U}$

The latter relation can be rewritten for any temperature $(T)$ using the parameters determined experimentally by DSC:

$\Delta G_{N-U}(T)=\Delta H_{c a l}\left(1-T / T_{m}\right)+\Delta C p\left(T-T_{m}\right)-T \Delta C p \ln \left(T / T_{m}\right)$

where $\Delta C p$ is the difference in heat capacity between the native and the unfolded state. Computing Eq. (3) in a temperature range where the native state prevails in solution provides the protein stability curve [56], i.e. the free energy of unfolding as a function of temperature (Fig. 10). In other words, this is the work required to disrupt the native state at any given temperature [57] and is also referred to as the thermodynamic stability. By definition, $\Delta G_{N-U}$ is zero at $T_{m}$, at equilibrium. At temperatures below $T_{m}$, the stability increases, as expected, but perhaps surprisingly for the non-specialist, the stability reaches a maximum then it decreases at lower temperatures (Fig. 10). In fact, this function predicts a temperature of cold-unfolding, which is generally not observed because it occurs below $0^{\circ} \mathrm{C}[58]$. Increasing the stability of a protein is essentially obtained by lifting the curve towards higher free energy values $[59,60]$, as exemplified by the hyperthermophilic protein (Fig. 10), whereas the low stability of a psychrophilic protein is reached by a global collapse of its curve. In all cases, there is no significant shift of the curves towards high or low temperatures. As far as extremophiles are concerned, one of the most puzzling observations is that most proteins obey this pattern, i.e. whatever the microbial source, either from hot springs or from sea ice, the maximal stability of their proteins is clustered around room temperature $[59,61,62]$. This indicates that the various and sometimes opposites forces driving folding are optimally balanced in this temperature range. It should be noted that when stability curves are computed from equilibrium unfolding by chemical denaturants, shifts of the temperature for maximal stability towards higher or lower temper- 


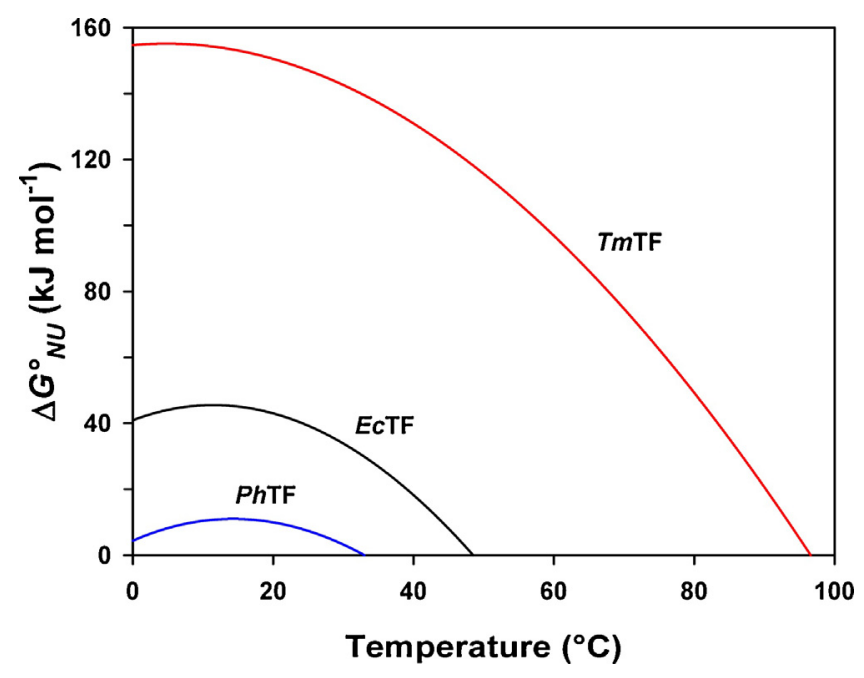

Fig. 10. Gibbs free energy of unfolding, or conformational stability, of homologous proteins. The work required to disrupt the native state is plotted as a function of temperature for psychrophilic (blue, PhTF), mesophilic (black, EcTF) and hyperthermophilic (red, TmTF) proteins.

Adapted from [18].

atures have been reported [24,63]. Such discrepancies remain to be clarified but possibly originate from the distinct unfolding mechanisms and the nature of the unfolded states. The $\Delta C p$ parameter in Eq. 3 contributes to the curvature of the stability curve and a decrease of this value induces a flattening of the function. Reduction of the $\Delta C p$ value has been reported for some thermophilic and hyperthermophilic proteins [18,23,64-66]. It has been shown that the difference in heat capacity between the native and unfolded states decreases with temperature and vanishes at around $120^{\circ} \mathrm{C}$ for most mesophilic proteins [67]. It follows that the higher the melting temperature, the lower the $\Delta C p$ value.

According to the bell-shaped stability curve, the environmental temperatures for mesophiles and hyperthermophiles lie on the right limb of the curve and obviously, the thermal dissipative force is used to promote molecular motions in these molecules. By contrast, the environmental temperatures for psychrophiles lie on the left limb of the stability curve. It follows that molecular motions in proteins at low temperatures are gained from the factors ultimately leading to cold-unfolding [49], i.e. the hydration of polar and non-polar groups and the weakening of the hydrophobic effect [68]. The origin of flexibility in psychrophilic proteins at low temperatures is therefore drastically different from mesophilic and hyperthermophilic proteins, the latter taking advantage of the conformational entropy rise with temperature to gain in mobility.

A surprising consequence of the free energy function for the psychrophilic protein shown in Fig. 10 is its weak stability at low temperatures when compared with mesophilic and thermophilic proteins, whereas it was intuitively expected that cold-adapted proteins should also be cold stable. This protein is in fact both heat and cold labile. As a result, cold unfolding of psychrophilic proteins has been experimentally recorded at the temperature predicted by the stability curve $[18,49]$, providing validation of the free energy function.

\section{Conclusions}

The literature on protein folding in temperature extremophiles is still scarce but this should improve rapidly because, besides fundamental aspects, the topic is significantly relevant in biotechnology, such as for the expression of soluble recombinant proteins at low temperature by psychrophiles [35] or for the synthesis of robust enzyme catalysts used in harsh industrial conditions [69]. The topic is also of interest for astrobiology as life might have evolved on cold or hot planets [70]. The available results underline the necessity of investigating series of homologous extremophilic and mesophilic proteins in order to screen the largest spectrum of biological temperatures. Current studies using extremophilic proteins which unfold fully reversibly according to a perfect twostate mechanism should refine the available data. Multidisciplinary approaches are also needed because, for instance, cold adaptation is not always the converse of hot adaptation. In addition, multiple factors can be involved as illustrated here by chaperones or by the effect of prolyl isomerization.

Finally, the main drawback in protein folding studies of extremophiles is the fact that the related experiments cannot be performed at the environmental temperatures. At low temperatures, condensation on optics strongly perturbs spectroscopic signals and requires abundant nitrogen flushing, which is not always technically feasible. At high temperatures, folding events become so fast that they cannot be recorded and all model protein substrates used in these experiments are mesophilic, such as GFP, and unfold or aggregate at temperatures well below those encountered by thermophiles. In all cases, results obtained in a limited window of temperatures, as well as the associated values, have to be extrapolated to either low or high temperatures, which can be controversial. Reviewers are sometimes reluctant to take these limitations into account.

\section{Acknowledgments}

I gratefully thank André Matagne (Liège University), Philipp Schmidpeter and Franz Schmid (Bayreuth University) for their expert scientific input in some of our works cited here. I also thank S. D'Amico for his earlier contribution, as well as previous PhD students, F. Piette, C. Struvay, A. Cipolla and A. Godin during their FRIA fellowship. Works at the author's laboratory were supported by the F.R.S-FNRS, Belgium (Fonds de la Recherche Fondamentale et Collective, contract numbers 2.4535.08, 2.4523.11 and U.N009.13) and by the Belgian program of Interuniversity Attraction Poles (iPros P7/44) initiated by the Federal Office for Scientific, Technical and Cultural Affairs.

\section{References}

[1] J.W. Deming, Psychrophiles and polar regions, Curr. Opin. Microbiol. 5 (2002) 301-309, S1369527402003296.

[2] K. Junge, H. Eicken, J.W. Deming, Bacterial Activity at -2 to -20 degrees $C$ in Arctic wintertime sea ice, Appl. Environ. Microbiol. 70 (2004) 550-557, http:// dx.doi.org/10.1128/AEM.70.1.550-557.2004.

[3] N.C. Mykytczuk, S.J. Foote, C.R. Omelon, G. Southam, C.W. Greer, L.G. Whyte, Bacterial growth at -15 degrees C; molecular insights from the permafrost bacterium Planococcus halocryophilus Or1, ISME J. 7 (2013) 1211-1226, http:// dx.doi.org/10.1038/ismej.2013.8.

[4] H. Latif, J.A. Lerman, V.A. Portnoy, Y. Tarasova, H. Nagarajan, A.C. Schrimpe-Rutledge, R.D. Smith, J.N. Adkins, D.H. Lee, Y. Qiu, K. Zengler, The genome organization of Thermotoga maritima reflects its lifestyle, PLoS Genet. 9 (2013) e1003485, http://dx.doi.org/10.1371/journal.pgen.1003485.

[5] G. Deckert, P.V. Warren, T. Gaasterland, W.G. Young, A.L. Lenox, D.E. Graham, R. Overbeek, M.A. Snead, M. Keller, M. Aujay, R. Huber, R.A. Feldman, J.M. Short, G.J. Olsen, R.V. Swanson, The complete genome of the hyperthermophilic bacterium Aquifex aeolicus, Nature 392 (1998) 353-358, http://dx.doi.org/10.1038/32831.

[6] E. Blochl, R. Rachel, S. Burggraf, D. Hafenbradl, H.W. Jannasch, K.O. Stetter, Pyrolobus fumarii, gen. and sp. nov., represents a novel group of archaea, extending the upper temperature limit for life to 113 degrees $C$, Extremophiles 1 (1997) 14-21, http://dx.doi.org/10.1007/s007920050010.

[7] K. Takai, K. Nakamura, T. Toki, U. Tsunogai, M. Miyazaki, J. Miyazaki, H. Hirayama, S. Nakagawa, T. Nunoura, K. Horikoshi, Cell proliferation at 122 degrees $\mathrm{C}$ and isotopically heavy $\mathrm{CH} 4$ production by a hyperthermophilic methanogen under high-pressure cultivation, Proc. Natl. Acad. Sci. U. S. A. 105 (2008) 10949-10954, http://dx.doi.org/10.1073/pnas.0712334105. 
[8] G. Feller, Protein stability and enzyme activity at extreme biological temperatures, J. Phys.-Condens. Mat. 22 (2010), http://dx.doi.org/10.1088/ 0953-8984/1022/1032/323101.

[9] G. Feller, Psychrophilic enzymes: from folding to function and biotechnology, Scientifica 512840 (2013), http://dx.doi.org/10.1155/2013/512840.

[10] G. Feller, C. Gerday, Psychrophilic enzymes: hot topics in cold adaptation, Nat. Rev. Microbiol. 1 (2003) 200-208, http://dx.doi.org/10.1038/nrmicro773.

[11] K.S. Siddiqui, R. Cavicchioli, Cold-adapted enzymes, Annu. Rev. Biochem. 75 (2006) 403-433, http://dx.doi.org/10.1146/annurev.biochem.75.103004. 142723.

[12] C. Struvay, G. Feller, Optimization to low temperature activity in psychrophilic enzymes, Int. J. Mol. Sci. 13 (2012) 11643-11665, http://dx.doi org/10.3390/ijms130911643.

[13] A. Kohen, R. Cannio, S. Bartolucci, J.P. Klinman, Enzyme dynamics and hydrogen tunnelling in a thermophilic alcohol dehydrogenase, Nature 399 (1999) 496-499, http://dx.doi.org/10.1038/20981.

[14] S. Kumar, R. Nussinov, How do thermophilic proteins deal with heat? Cell. Mol. Life Sci. 58 (2001) 1216-1233, http://dx.doi.org/10.1007/PL00000935.

[15] C. Vieille, G.J. Zeikus, Hyperthermophilic enzymes: sources, uses, and molecular mechanisms for thermostability, Microbiol. Mol. Biol. Rev. 65 (2001) 1-43, http://dx.doi.org/10.1128/MMBR.65.1.1-43.2001.

[16] A. Hoffmann, B. Bukau, G. Kramer, Structure and function of the molecular chaperone Trigger Factor, Biochim. Biophys. Acta 2010 (1803) 650-661, http://dx.doi.org/10.1016/j.bbamcr.2010.01.017.

[17] C. Medigue, E. Krin, G. Pascal, V. Barbe, A. Bernsel, P.N. Bertin, F. Cheung, S Cruveiller, S. D’Amico, A. Duilio, G. Fang, G. Feller, C. Ho, S. Mangenot, G. Marino, J. Nilsson, E. Parrilli, E.P. Rocha, Z. Rouy, A. Sekowska, M.L. Tutino, D. Vallenet, G. von Heijne, A. Danchin, Coping with cold: the genome of the versatile marine Antarctica bacterium Pseudoalteromonas haloplanktis TAC125, Genome Res. 15 (2005) 1325-1335, http://dx.doi.org/10.1101/gr. 4126905.

[18] C. Struvay, S. Negro, A. Matagne, G. Feller, Energetics of protein stability at extreme environmental temperatures in bacterial trigger factors, Biochemistry 52 (2013) 2982-2990, http://dx.doi.org/10.1021/bi4002387.

[19] D. Mitsuya, S. Tanaka, H. Matsumura, N. Urano, K. Takano, K. Ogasahara, M. Takehira, K. Yutani, M. Ishida, Strategy for cold adaptation of the tryptophan synthase alpha subunit from the psychrophile Shewanella frigidimarina K14-2: crystal structure and physicochemical properties, J. Biochem. 155 (2014) 73-82, http://dx.doi.org/10.1093/jb/mvt098.

[20] A.A. Nickson, J. Clarke, What lessons can be learned from studying the folding of homologous proteins? Methods 52 (2010) 38-50, http://dx.doi.org/10. 1016/j.ymeth.2010.06.003.

[21] D. Perl, C. Welker, T. Schindler, K. Schroder, M.A. Marahiel, R. Jaenicke, F.X. Schmid, Conservation of rapid two-state folding in mesophilic, thermophilic and hyperthermophilic cold shock proteins, Nat. Struct. Biol. 5 (1998) 229-235, PMID: 9501917

[22] T.B. Topping, L.M. Gloss, Stability and folding mechanism of mesophilic, thermophilic and hyperthermophilic archael histones: the importance of folding intermediates, J. Mol. Biol. 342 (2004) 247-260, http://dx.doi.org/10. 1016/j.jmb.2004.07.045.

[23] M. Wallgren, J. Aden, O. Pylypenko, T. Mikaelsson, L.B. Johansson, A. Rak, M. Wolf-Watz, Extreme temperature tolerance of a hyperthermophilic protein coupled to residual structure in the unfolded state, J. Mol. Biol. 379 (2008) 845-858, http://dx.doi.org/10.1016/j.jmb.2008.04.007.

[24] K.A. Luke, C.L. Higgins, P. Wittung-Stafshede, Thermodynamic stability and folding of proteins from hyperthermophilic organisms, FEBS J. 274 (2007) 4023-4033, http://dx.doi.org/10.1111/j.1742-4658.2007.05955.x.

[25] A. Mukaiyama, K. Takano, Slow unfolding of monomeric proteins from hyperthermophiles with reversible unfolding, Int. J. Mol. Sci. 10 (2009) 1369-1385, http://dx.doi.org/10.3390/ijms10031369.

[26] K. Ogasahara, M. Nakamura, S. Nakura, S. Tsunasawa, I. Kato, T. Yoshimoto, K. Yutani, The unusually slow unfolding rate causes the high stability of pyrrolidone carboxyl peptidase from a hyperthermophile, Pyrococcus furiosus: equilibrium and kinetic studies of guanidine hydrochloride-induced unfolding and refolding, Biochemistry 37 (1998) 17537-17544, http://dx.doi. org/10.1021/bi9814585.

[27] J. Okada, T. Okamoto, A. Mukaiyama, T. Tadokoro, D.J. You, H. Chon, Y. Koga, K. Takano, S. Kanaya, Evolution and thermodynamics of the slow unfolding of hyperstable monomeric proteins, BMC Evol. Biol. 10 (2010) 207, http://dx.doi. org/10.1186/1471-2148-10-207.

[28] B.A. Kelch, D.A. Agard, Mesophile versus thermophile: insights into the structural mechanisms of kinetic stability, J. Mol. Biol. 370 (2007) 784-795, http://dx.doi.org/10.1016/j.jmb.2007.04.078.

[29] P. Wittung-Stafshede, Slow unfolding explains high stability of thermostable ferredoxins: common mechanism governing thermostability? Biochim. Biophys. Acta 2004 (1700) 1-4, http://dx.doi.org/10.1016/j.bbapap.2004.04. 002 .

[30] X. Du, P. Sang, Y.L. Xia, Y. Li, J. Liang, S.M. Ai, X.L. Ji, Y.X. Fu, S.Q. Liu, Comparative thermal unfolding study of psychrophilic and mesophilic subtilisin-like serine proteases by molecular dynamics simulations, J. Biomol. Struct. Dyn. 35 (2017) 1500-1517, http://dx.doi.org/10.1080/07391102.2016. 1188155.

[31] A. Cipolla, S. D’Amico, R. Barumandzadeh, A. Matagne, G. Feller, Stepwise adaptations to low temperature as revealed by multiple mutants of psychrophilic alpha-amylase from Antarctic bacterium, J. Biol. Chem. 286 (2011) 38348-38355, http://dx.doi.org/10.1074/jbc.M111.274423.
[32] H. Dong, A. Mukaiyama, T. Tadokoro, Y. Koga, K. Takano, S. Kanaya, Hydrophobic effect on the stability and folding of a hyperthermophilic protein, J. Mol. Biol. 378 (2008) 264-272, http://dx.doi.org/10.1016/j.jmb. 2008.02.039.

[33] A. Godin-Roulling, P.A. Schmidpeter, F.X. Schmid, G. Feller, Functional adaptations of the bacterial chaperone trigger factor to extreme environmental temperatures, Environ. Microbiol. 17 (2015) 2407-2420, http://dx.doi.org/10.1111/1462-2920.12707.

[34] J. King, C. Haase-Pettingell, A.S. Robinson, M. Speed, A. Mitraki, Thermolabile folding intermediates: inclusion body precursors and chaperonin substrates, FASEB J. 10 (1996) 57-66, PMCID: PMC2040114.

[35] M. Giuliani, E. Parrilli, F. Sannino, G. Apuzzo, G. Marino, M.L. Tutino, Soluble recombinant protein production in Pseudoalteromonas haloplanktis TAC125, Methods Mol. Biol. 1258 (2015) 243-257, http://dx.doi.org/10.1007/978-14939-2205-5_13.

[36] R. Suno, H. Taguchi, R. Masui, M. Odaka, M. Yoshida, Trigger factor from Thermus thermophilus is a $\mathrm{Zn}^{2+}$-dependent chaperone, J. Biol. Chem. 279 (2004) 6380-6384, http://dx.doi.org/10.1074/jbc.M311572200.

[37] F.U. Hartl, M. Hayer-Hartl, Converging concepts of protein folding in vitro and in vivo, Nat. Struct. Mol. Biol. 16 (2009) 574-581, http://dx.doi.org/10.1038/ nsmb.1591.

[38] A.T. Large, M.D. Goldberg, P.A. Lund, Chaperones and protein folding in the archaea, Biochem. Soc. Trans. 37 (2009) 46-51, http://dx.doi.org/10.1042/ BST0370046.

[39] R.L. Baldwin, The search for folding intermediates and the mechanism of protein folding, Annu. Rev. Biophys. 37 (2008) 1-21, http://dx.doi.org/10. 1146/annurev.biophys.37.032807.125948.

[40] F.X. Schmid, Prolyl isomerization in protein folding, in: J. Buchner, T. Kiefhaber (Eds.), Protein Folding Handbook, Wiley-VCH, Weinheim, 2005, pp. 916-945, http://dx.doi.org/10.1002/9783527619498.ch25.

[41] G. Zoldak, T. Aumuller, C. Lucke, J. Hritz, C. Oostenbrink, G. Fischer, F.X. Schmid, A library of fluorescent peptides for exploring the substrate specificities of prolyl isomerases, Biochemistry 48 (2009) 10423-10436, http://dx.doi.org/10.1021/bi9014242.

[42] K. Watanabe, T. Masuda, H. Ohashi, H. Mihara, Y. Suzuki, Multiple proline substitutions cumulatively thermostabilize Bacillus cereus ATCC7064 oligo-1, 6-glucosidase. Irrefragable proof supporting the proline rule, Eur. J. Biochem. 226 (1994) 277-283, http://dx.doi.org/10.1111/j.1432-1033.1994.tb20051.x.

[43] C. Scholz, G. Stoller, T. Zarnt, G. Fischer, F.X. Schmid, Cooperation of enzymatic and chaperone functions of trigger factor in the catalysis of protein folding, EMBO J. 16 (1997) 54-58, http://dx.doi.org/10.1093/emboj/16.1.54.

[44] T. Maruyama, R. Suzuki, M. Furutani, Archaeal peptidyl prolyl cis-trans isomerases (PPIases) update 2004, Front. Biosci. 9 (2004) 1680-1720, http:// dx.doi.org/10.2741/1361

[45] S. D'Amico, J.C. Marx, C. Gerday, G. Feller, Activity-stability relationships in extremophilic enzymes, J. Biol. Chem. 278 (2003) 7891-7896, http://dx.doi. org/10.1074/jbc.M212508200.

[46] S.T. Ladani, M.G. Souffrant, A. Barman, D. Hamelberg, Computational perspective and evaluation of plausible catalytic mechanisms of peptidyl-prolyl cis-trans isomerases, Biochim. Biophys. Acta 2015 (1850) 1994-2004, http://dx.doi.org/10.1016/j.bbagen.2014.12.023.

[47] F. Piette, S. D’Amico, C. Struvay, G. Mazzucchelli, J. Renaut, M.L. Tutino, A. Danchin, P. Leprince, G. Feller, Proteomics of life at low temperatures: trigger factor is the primary chaperone in the Antarctic bacterium Pseudoalteromonas haloplanktis TAC125, Mol. Microbiol. 76 (2010) 120-132, http://dx.doi.org/10. 1111/j.1365-2958.2010.07084.x.

[48] F. Piette, C. Struvay, G. Feller, The protein folding challenge in psychrophiles: facts and current issues, Environ. Microbiol. 13 (2011) 1924-1933, http://dx. doi.org/10.1111/j.1462-2920.2011.02436.x.

[49] G. Feller, D. d'Amico, C. Gerday, Thermodynamic stability of a cold-active alpha-amylase from the Antarctic bacterium Alteromonas haloplanctis, Biochemistry 38 (1999) 4613-4619, http://dx.doi.org/10.1021/bi982650+

[50] T. Thomas, R. Cavicchioli, Effect of temperature on stability and activity of elongation factor 2 proteins from Antarctic and thermophilic methanogens, J. Bacteriol. 182 (2000) 1328-1332, http://dx.doi.org/10.1128/JB.182.5.13281332.2000.

[51] T. Collins, M.A. Meuwis, C. Gerday, G. Feller, Activity, stability and flexibility in glycosidases adapted to extreme thermal environments, J. Mol. Biol. 328 (2003) 419-428, http://dx.doi.org/10.1016/S0022-2836(03)00287-0.

[52] D. Georlette, B. Damien, V. Blaise, E. Depiereux, V.N. Uversky, C. Gerday, G. Feller, Structural and functional adaptations to extreme temperatures in psychrophilic, mesophilic, and thermophilic DNA ligases, J. Biol. Chem. 278 (2003) 37015-37023, http://dx.doi.org/10.1074/jbc.M305142200.

[53] A. Cipolla, F. Delbrassine, J.L. Da Lage, G. Feller, Temperature adaptations in psychrophilic, mesophilic and thermophilic chloride-dependent alpha-amylases, Biochimie 94 (2012) 1943-1950, http://dx.doi.org/10.1016/j. biochi.2012.05.013.

[54] D.M. LeMaster, J. Tang, G. Hernandez, Absence of kinetic thermal stabilization in a hyperthermophile rubredoxin indicated by 40 microsecond folding in the presence of irreversible denaturation, Proteins 57 (2004) 118-127, http://dx. doi.org/10.1002/prot.20181.

[55] M. Sawano, H. Yamamoto, K. Ogasahara, S. Kidokoro, S. Katoh, T. Ohnuma, E. Katoh, S. Yokoyama, K. Yutani, Thermodynamic basis for the stabilities of three CutA1s from Pyrococcus horikoshii, Thermus thermophilus, and Oryza sativa, with unusually high denaturation temperatures, Biochemistry 47 (2008) 721-730, http://dx.doi.org/10.1021/bi701761m. 
[56] W.J. Becktel, J.A. Schellman, Protein stability curves, Biopolymers 26 (1987) 1859-1877, http://dx.doi.org/10.1002/bip.360261104.

[57] P.L. Privalov, Stability of proteins: small globular proteins, Adv. Protein Chem. 33 (1979) 167-241, PMID: 44431.

[58] P.L. Privalov, Cold denaturation of proteins, Crit. Rev. Biochem. Mol. Biol. 25 (1990) 281-305, http://dx.doi.org/10.3109/10409239009090613.

[59] S. Kumar, R. Nussinov, Experiment-guided thermodynamic simulations on reversible two-state proteins: implications for protein thermostability, Biophys. Chem. 111 (2004) 235-246, http://dx.doi.org/10.1016/j.bpc.2004.06. 005.

[60] A. Razvi, J.M. Scholtz, Lessons in stability from thermophilic proteins, Protein Sci. 15 (2006) 1569-1578, http://dx.doi.org/10.1110/ps.062130306.

[61] D.C. Rees, A.D. Robertson, Some thermodynamic implications for the thermostability of proteins, Protein Sci. 10 (2001) 1187-1194, http://dx.doi. org/10.1110/ps.180101.

[62] S. Kumar, C.J. Tsai, R. Nussinov, Maximal stabilities of reversible two-state proteins, Biochemistry 41 (2002) 5359-5374, http://dx.doi.org/10.1021/ bi012154c.

[63] O. Garcia-Arribas, R. Mateo, M.M. Tomczak, P.L. Davies, M.G. Mateu, Thermodynamic stability of a cold-adapted protein, type III antifreeze protein, and energetic contribution of salt bridges, Protein Sci. 16 (2007) 227-238, http://dx.doi.org/10.1110/ps.062448907.

[64] S. Knapp, A. Karshikoff, K.D. Berndt, P. Christova, B. Atanasov, R. Ladenstein, Thermal unfolding of the DNA-binding protein Sso7d from the hyperthermophile Sulfolobus solfataricus, J. Mol. Biol. 264 (1996) 1132-1144, http://dx.doi.org/10.1006/jmbi.1996.0701.
[65] B.S. McCrary, S.P. Edmondson, J.W. Shriver, Hyperthermophile protein folding thermodynamics: differential scanning calorimetry and chemical denaturation of Sac7d, J. Mol. Biol. 264 (1996) 784-805, http://dx.doi.org/10. 1006/jmbi.1996.0677.

[66] S. Robic, M. Guzman-Casado, J.M. Sanchez-Ruiz, S. Marqusee, Role of residual structure in the unfolded state of a thermophilic protein, Proc. Natl. Acad. Sci. U. S. A. 100 (2003) 11345-11349, http://dx.doi.org/10.1073/pnas. 1635051100.

[67] P.L. Privalov, A.I. Dragan, Microcalorimetry of biological macromolecules, Biophys. Chem. 126 (2007) 16-24, http://dx.doi.org/10.1016/j.bpc.2006.05. 004.

[68] G.I. Makhatadze, P.L. Privalov, Energetics of protein structure, Adv. Protein. Chem. 47 (1995) 307-425, PMID: 8561051

[69] M.S. Urbieta, E.R. Donati, K.G. Chan, S. Shahar, L.L. Sin, K.M. Goh, Thermophiles in the genomic era: biodiversity, science, and applications, Biotechnol. Adv. 33 (2015) 633-647, http://dx.doi.org/10.1016/j.biotechadv.2015.04.007.

[70] C. Moissl-Eichinger, C. Cockell, P. Rettberg, Venturing into new realms? Microorganisms in space, FEMS Microbiol. Rev. 40 (2016) 722-737, http://dx. doi.org/10.1093/femsre/fuw015. 